\title{
Guest editorial: Special issue on ECRTS 2008
}

\author{
Alan Burns \\ Published online: 2 July 2009 \\ (C) Springer Science+Business Media, LLC 2009
}

As Michael Gonzalez Harbour said in the introduction to the special issue for ECRTS 2007, "embedded systems have an increasing influence in technology and in the society in general, with a growing number of application fields and expanding capabilities and complexity. These systems usually control or monitor artefacts and elements of the outside world that change with time. As a consequence, most embedded systems have requirements to adapt the timing of their response to the changes of their environment, which leads to the need for using real-time techniques and methods in their design, implementation, and testing. Many embedded systems today have increasingly high levels of criticality as they control industrial processes, vehicles, telecommunications, or medical equipment. This poses important challenges to the real-time systems community in our path towards finding better methods and techniques that can be used to effectively engineer safe and reliable embedded systems."

In 2008 ECRTS (Euromicro/European Conference on Real-Time Systems) celebrated its 20th conference. This series of meetings has grown from a relatively small workshop to a top quality international conference that can be proud of the contribution it makes to the development of real-time technologies and theory. The location for the 2008 event was Prague in the Czech Republic, a city that itself has changed considerable over the last twenty years. The conference and the venue were both a great success.

Four of the many excellent papers presented at the conference were selected by the programme committee for inclusion in this special issue. Each paper was extended to add significant extra material and the resulting papers were re-reviewed and subject to

\footnotetext{
A. Burns $(\varangle)$

Department of Computer Science, University of York, Heslington, York YO10 5DD, UK

e-mail: alan.burns@cs.york.ac.uk

url: http://www.cs.york.ac.uk/ burns/
} 
the usual editorial process. The result is a special issue that I hope will be of interest to the real-time community.

Reflecting the current focus of much research in real-time systems, three of the four papers address issues pertinent to multiprocessor platforms. The first paper: "An analysis of global EDF schedulability for arbitrary-deadline sporadic task systems" by Baker and Baruah, is concerned with the fundamental question of schedulability for EDF systems executing on multiprocessor platform when tasks can migrate between processors at run-time. They extend previous results that constrained the deadline of each task to be less than period.

The second paper: "Optimal virtual cluster-based multiprocessor scheduling" by Easwaran, Shin and Lee, investigates a middle ground between partitioned and global allocation of tasks. They propose a clustering approach that allows some level of migration (within a cluster) but not full global dispatching. This theme of adding structure to the behaviour of the application's tasks is continued in the paper by Leontyev and Anderson entitled, "A hierarchical multiprocessor bandwidth reservation scheme with timing guarantees". Here a multiprocessor scheduling scheme is presented that supports hierarchical containers that encapsulate groups of tasks (and other containers). They support soft real-time tasks, but deliver a guaranteed upper bound on completion.

The final paper, the original of which won the prize for best paper at the conference, presents a novel way of attempting to improve the quality of service for realtime tasks. The paper: "A gravitational task model with arbitrary anchor points for target sensitivity real-time applications" is by Guerra and Fohler. The paper presents a task model based on a gravitational analogy; this allows a task to express its preference as to when, in its execution window, it would ideally complete. 